\title{
Explaining Unification in Physics Internally
}

\author{
Kian Salimkhani*†
}

\begin{abstract}
In this paper I challenge two widespread convictions about unification in physics: (1) unification is an aim of physics and (2) unification is driven by metaphysical or metatheoretical presuppositions. I call these external explanations of why there is unification in physics. Against this, I claim that (a) unification is a by-product of physical research and (b) unification is driven by basic methodological strategies of physics alone (without any appeal to metaphysical or metatheoretical presuppositions). I call this an internal (or methodological) explanation of why there is unification in physics. To support my claims, I will investigate the actual practice undertaken in physics in paradigmatic examples of unification.
\end{abstract}

Keywords: Unification; Methodology; Physics; Metaphysics

\section{Introduction}

In this paper I challenge two common convictions about unification in physics: (1) unification is an aim (or the aim) of physics, and (2) unification is driven by metaphysical or metatheoretical presuppositions. Against this, I claim that (a) unification is a result - or for dialectical effect: a by-product - of physical research, and (b) unification is driven by basic methodological strategies of physics alone. In particular, I claim that unification is not driven by any appeal to metaphysical or metatheoretical presuppositions. Accordingly, the main objective of this paper is to answer the following questions: If we take physics as not explicitly aiming for unification, then how do we explain the many apparent cases of unification in physics, i.e., why is there a unificatory practice in physics (or more specifically: why are there certain unificatory research programmes); and, what is the methodological 'toolbox' that brings about unification, if metaphysical or metatheoretical presuppositions are not responsible?

Formally speaking (building on Salimkhani (2018)), there are two options for responding to the first question: either we explain unification in physics

${ }^{*}$ This work was supported by the German Research Foundation (DFG), grant number: FOR 2495 (research unit Inductive Metaphysics).

${ }^{\dagger}$ This is a post-peer-review, pre-copyedit version of an article published in Synthese. The final authenticated version is available online at: http://dx.doi.org/10.1007/ s11229-019-02436-x. 
internally or externally. An explanation is internal if it is based on the basic (or genuine) aims and methods of physics only - we may say in short: an internal explanation is based on physical reasoning. On the other hand, an explanation is external if it is based on additional presuppositions, aims, and methods that cannot be considered an essential part of physics (e.g., metaphysical presuppositions) — we may say in short: an external explanation is based on general philosophical reasoning.

Take the basic (or genuine) methodology of physics to be the methodology that is associated with (or conducive for) a minimal set of aims that physics necessarily sets for itself and that defines the discipline. Certainly, physics is a sufficiently directed enterprise to be expected to have such a set of aims, but admittedly there is no consensus on what aims to include (e.g., Hüttemann (1997)). Still, with respect to physics practice I propose to take the following two as the most parsimonious, sufficiently accepted, and central aims that need to be accounted for in one way or another: empirical adequacy and theoretical consistency. Physics is expected, first and foremost, to yield empirically adequate theories, i.e. correctly represent the phenomena and empirical data. Also, physical theories are expected to be sufficiently consistent to not include contradictory statements - at least not within a particular theory. Assuming that physics specifically aims at unification, on the other hand, is a stronger and, hence, more controversial claim. In particular, if unification is not plainly conceived of as an additional aim, it involves some argument regarding how empirical adequacy is taken care of. ${ }^{1}$ In both cases, the position is more costly than the minimal set proposed.

This being said, it is important to emphasise that I am not engaging in the project of arguing in favour of some specific set of aims here. I do agree that this issue is rather involved. In particular, it may be possible to do without some versions of theoretical consistency, ${ }^{2}$ or expect theoretical consistency to be reducible to empirical adequacy. ${ }^{3}$ Ultimately, it is not so much the aims, but the concrete methodology that matters (see below). What I am after in this paper is that for explaining unification in physics it is sufficient to appeal to a 'down-to-earth' conception of physics that gets rid of as many controversial aims, background assumptions (e.g., some elaborated theory of explanation), and methods as possible: already a deflationary understanding of physics' (internal) aims and methodological strategies is able to explain its unificatory practice.

In summary, the internal view - which I argue for in this paper-holds that those methodological strategies that are an essential part of doing everyday research in physics (e.g., inductive generalisations or resolving theoretical incon-

\footnotetext{
${ }^{1}$ The aim of theoretical consistency is straightforwardly absorbed in the stronger aim of unification (see also section 2), but empirical adequacy is not.

${ }^{2}$ See Crowther and Linnemann (2018).

${ }^{3}$ See Steinberger (2017) with respect to general issues of rationality: "The thought is that any instance of my violating (NC) [the requirement to not both believe $A$ and $\neg A$ ] is eo ipso an instance in which my beliefs are out of whack with the evidence. For when I hold contradictory beliefs, at least one of the beliefs must be unsupported by the evidence." I thank Stefan Heidl for pointing this out to me.
} 
sistencies) are also responsible for physics' unificatory practice. Accordingly, already a minimal understanding of physics' aims and methods is headed towards unification. Against this, the external view holds either that there is a unificatory practice in physics because unification is what physics explicitly aims for, or that attempts at unification are based on metaphysical or metatheoretical presuppositions (i.e., philosophical reasoning). Hence, for the external view, unification is the (imposed) result of additionally constraining research by assumptions that are not part of physics proper.

To argue for the external view, one would have to show that the unificatory practice in physics hinges on certain external (e.g., metaphysical or metatheoretical) presuppositions or an explicit aiming for unification that is methodologically active. In particular, one would have to demonstrate where and how physicists do (and need to) employ external reasoning to justify research programmes (at least with respect to their pursuit-worthiness) and to methodologically account for unification. On the contrary, to argue for the internal view, one has to show that essential methodological strategies of physics produce a more unified picture as a by-product. In short, explaining why and specifying in which sense physics is about unification amounts to investigating its actual practice. Accordingly, I will analyse paradigmatic examples of unification to support my claims.

As philosophical debates on unification usually focus on other aspects, I take it that the main questions of this paper are not yet sufficiently addressed nor satisfyingly answered in the literature. ${ }^{4}$ Nevertheless, I propose to take the following as typical philosophical explanations of the unificatory practice in physics: (1) claiming that physics generally aims at unification (for an overview see Hüttemann (1997), for example) or other epistemic values like explanation, with explanation being unification (drawing on Friedman (1974) and Kitcher (1981), for example) or being supported by unification (see Salmon (1984) and Salmon (1990)); or (2) claiming that we can identify specific metaphysical (e.g., 'unity of nature') or metatheoretical (e.g., simplicity or 'beauty' of mathematical representation) presuppositions that guide and drive scientific research towards unification (e.g., Mattingly (2005) and Hossenfelder (2018); see also Cat (1998) reviewing the history of debates on unification among physicists).

How should these explanations be classified with respect to the internalexternal distinction? First, proponents of the view that unification is an aim of physics must argue either that the genuine methodology of physics associated with the minimal set of aims is sufficient to achieve this additional aim or that it is not. ${ }^{5}$ The former is compatible with the internal view proposed in this paper. Unification is then ultimately conceived of as a redundant aim: the genuine methodology that supports the indispensable aims (empirical adequacy and

\footnotetext{
${ }^{4}$ In Salimkhani (2018), I provide an analysis of unification in the context of quantum gravity research that is in line with this paper (see also section 3.4), but do not generalise the findings or clarify key notions such as 'internal' and 'external' unification.

${ }^{5}$ This ambiguity is usually not acknowledged in the literature and stems from the fact that such positions typically remain silent on how (aiming at) unification is obtained methodologically.
} 
theoretical consistency) also brings about unification as a by-product. ${ }^{6}$ This is the reason why heuristic readings like Kao's (2017) are generally in accordance with an internal understanding - although they typically do not acknowledge the redundancy. On the contrary, denying that the minimal set of aims is sufficient basically collapses into claim (2): physics proper is taken to be guided and constrained by aiming at unification - or rather by certain additional methodologically active presuppositions associated with this aim.

While I accept - albeit not without criticism - that there is a certain way of speaking about physics 'aiming at unification' which is generally compatible with the internal view (and may especially fuel readings in terms of heuristics), I contest the external version of this claim. In this sense, the paper also explicates how we should understand the 'aiming at unification' talk so that it is in accordance with physics practice.

Second, claiming that metaphysical or metatheoretical presuppositions guide and drive scientific research towards unification explicitly adopts external reasoning: physics is taken to pursue a (specifically enriched) methodology which is biased towards a striving for unification by including metaphysical or metatheoretical presuppositions (call this a 'unificationist methodology'). The reasoning is that unification in physics is driven by presuppositions that arguably are not part of physics proper; for example, unification could be driven by some metaphysical presupposition like 'there is unity in nature' or 'everything is quantum'. Arguing that it is such additional presuppositions that bring about unification in physics seems to imply that there is (or could be) a less unificatorily biased way of doing physics (i.e., the basic methodology without such additional presuppositions) which could not account for the unificatory practice. I will argue that this is a misleading characterisation of how physics works.

One has to admit, however, that at first sight external explanations do seem justified by the long history of successful and failed attempts at unification, and by the fact that many physicists do subscribe to the view that finding a unified theory encompassing all physical phenomena is what physics ultimately aims at. Additionally, talk about unification is usually not merely descriptive, but rather a normative claim regarding what physics is about and how physics should proceed (in the future): Specific attempts at unification may fail, but nevertheless it is the way to go. As Maudlin (1996) puts it:

Today, anyone inquiring [...] into the current status of fundamental physical theory is virtually guaranteed to be told the following tale. In the first part of this century, physicists had verified the existence of four basic physical forces [...]. Passably accurate theories of these forces individually have been developed, but those theories do not yet demonstrate any deep connection [...]. The aim of physics is now to produce theories which unify these forces, which show, ultimately, that there is at base only one fundamental force in

\footnotetext{
${ }^{6}$ This understanding trivialises the claim that physics aims at unification: After all, unification is reduced to the status of being the result of other aims - so why should we consider it an aim? Why should we hold on to this ambiguous way of speaking?
} 
the universe $[\ldots]$. The first step in this program has already been taken [...] [t]he other steps, though still to be achieved, have already been named $[\ldots]$. This image of the future course of physical theory has become so pervasive as to rank almost as dogma. (Maudlin, 1996, 129)

According to this 'physicist's tale', unification exhibits the status of a methodological "commitment" that "puts rather strong constraints" on physics (Maudlin, 1996, 129f). This interpretation of physics practice might raise the following worry: physics (or rather mainstream research programmes in physics) might be led astray by the 'dogma of unification' and eventually run into a dead end, since physicists do not even consider the possibility of a disunited picture anymore - a worry resembling Wolfgang Pauli's verdict on Einstein's unified field theory: "What God put apart, no one shall unify." 7 I intend to assuage such worries by demonstrating that, in fact, unification is obtained indirectly by essential parts of the physics methodology, rendering unification a natural consequence of doing physics.

As mentioned, one might also argue (without appeal to metaphysics) that unification should be understood as a heuristic strategy for guiding theory development-based on the meta-inductive observation that it previously had proven worthwhile to explore directions of research that are assumed to exhibit a unifying power. Kao (2017) can be taken to present such a position. ${ }^{8}$

It strikes me that both readings Maudlin's physicist's tale and Kao's heuristics miss important insights in how theory development in physics is obtained methodologically. Moreover, without any need, such readings pave the way for criticism similar to, for example, Hossenfelder (2018). In short, there is more to learn.

As a science, modern physics subsumes the diversity of singular events under general laws of nature. To allow for this, physics arguably needs to employ some fundamental assumptions about its subject matter, but unification (of these laws of nature), so I claim, does not depend on any additional metaphysical or metatheoretical presuppositions that are not already implicit in genuine physics strategies themselves. It is not the case that we forcefully try to unify what God put apart. Rather, whenever we try to understand a limited domain we are pushed beyond its boundaries. In the course of physical research the concrete objects of research that physicists are concerned with contingently prove to exhibit surprisingly substantial links to other objects of research as

\footnotetext{
${ }^{7}$ My translation of "Was Gott getrennt hat, soll der Mensch nicht vereinen" (Treder, 1983).

${ }^{8}$ I do not argue against Kao's proposal, as I take her reading of unification playing some role in heuristics to be weak enough to be compatible with what I try to put forward in this paper. In fact, I agree with Kao on what physicists are actually concerned with (essentially also diminishing the alleged heuristic role of unification): "[...] indeed, it does not even seem to be the case that finding an overarching theory was the primary motivation to work in the various domains. [...] it is worth noting that this is the opposite of what we might think would be the case if the goal were, for instance, to find unifying theories because unifying explanations are a crucial characteristic of theories. Instead, it seems that the primary motivation was to solve existing problems in each scientific domain" (Kao, 2017). My project is that of taking this insight seriously.
} 
a matter of fact. So, we may say that the objects of research do 'effectively' turn out to fit together in a manner rather similar to jigsaw pieces. But to say this is not to presuppose the unity of nature. ${ }^{9}$ Hence, the claim is that even something as speculative as the quest for a theory of quantum gravity (QG) should be understood as the result of an immanent analysis of our theoretically most advanced and experimentally best-tested framework (see section 3.4), not as a striving for unification simpliciter. Accordingly, unification results as an epiphenomenon, a by-product, of exploiting genuine scientific methods. Philosophy should not obscure this important characteristic of physics by the usual talk about the alleged striving for unification.

In the following, after a few general remarks on unification, the internalexternal distinction is carved out in section 2. Afterwards, the practice of unification is explored in more detail with respect to four instructive examples in section 3 to support my claims. I conclude by briefly revisiting the resulting perspective on unification in section 4, adding some comments on how these findings can be useful for metaphysics.

\section{Mapping Concepts of Unification}

Usually, talk about unification in physics is not about unifying language or terminology, but something more substantial like 'unifying different laws of nature' (which means something like 'generalising or subsuming the respective laws into some superordinated theory in a meaningful way'). For example, Galileo's and Kepler's laws are 'unified' in Newton's universal law of gravitation, because Newton's law comprises and explains Galileo's and Kepler's laws. This is a tentative explication of how unification reduces plurality and reveals a typically unforeseen affinity between its objects. But what are the exact criteria for calling something 'unification'?

Trying to pin down such criteria, Maudlin (1996) distinguishes different levels with respect to their strength (with only the highest level amounting to unification). ${ }^{10}$ Maudlin argues that the following conditions are important but insufficient for saying that two laws of nature are 'unified': mere 'consistency', sharing a 'common explanatory structure', and exhibiting some 'nomic connection'. "The fact that a theory of embryonic development does not contradict a theory of the formation of the rings of Saturn" does not render the two unified (Maudlin, 1996, 130), neither does a common explanatory structure that does not put any constraint on the explanantia (Maudlin, 1996, 131). A theory that combines Newton's law of gravitation and Coulomb's law of electricity, for example, offers a common explanatory structure for electricity and gravi-

\footnotetext{
${ }^{9}$ As a position which refrains from metaphysical presuppositions in explaining the tendency towards unification, the internal perspective starts neither from a unitist's (e.g., von Weizsäcker (1980)) nor from a disunitist's (e.g., Cartwright (1999)) view —insisting on either 'unity' or 'disunity' in nature. Still, ultimately, we may favour one view over the other by help of inference to the best explanation (see also section 4).

${ }^{10}$ I shall assume that Maudlin's general picture is accepted albeit it with minor qualifications (see, for example, Morrison (2000), and Rickles and French (2006)).
} 
tation, but since "the forces are not postulated to have anything in particular to do with one another" this is not "unifying' gravity and electricity (Maudlin, 1996, 131). Instead, Maudlin insists that the notion of unification requires that ontological entities or structures are unified. This is why electromagnetism in special relativity (SR) exemplifies the strongest case for Maudlin: Here, the electric and magnetic fields are no longer fundamental entities of the theory, but are "replaced by" some underlying structure represented by the electromagnetic field tensor (Maudlin, 1996, 131-133). So, the highest level, i.e., unification, is obtained when previously independent entities (or structures) are reduced to a single entity (or structure) in the new theory.

Overall, I agree with Maudlin's classification; this paper, however, is focused on another issue, namely investigating the methodological aspects in the process of unification (that is the process of climbing Maudlin's conceptual ladder starting from the diversity of the phenomena and ending - maybe - in ontological unification). This objective entails that conceptual analysis cannot do the job, but the actual practice of physics and its methodological 'toolbox' (or 'mechanism') need to be investigated. For this, I propose to map different concepts of unification by asking two questions: (1) What is the object of unification?; and, (2) How does unification work methodologically?

The first question seems easy to answer. There are three levels (or dimensions) of unification: (1.1) We can obtain a phenomenological (or nomological) unification at the level of phenomenological laws of nature like Kepler's laws. Here, a variety of phenomena is nomologically unified by some law of nature. (1.2) Unification can be about (already existing) theoretical structure, e.g. physical theories. Call this theoretical unification. (1.3) We can obtain unification at the level of ontology, i.e., ontological unification, where ontic entities or structures are unified. For example, in the case of SR the electric and magnetic fields are unified to the electromagnetic field. Note that while unifying ontology generally requires theoretical and phenomenological unification, the opposite is not true. So, the strength of the unification obtained is understood to increase from (1.1) to (1.3).

Concerning the second question on how unification is actually worked out, I propose to distinguish five methodological strategies that physics regularly employs and that bring about a unificatory impact (as will be further discussed when considering the case studies in the next section): (2.1) inductive generalisation from phenomena to (phenomenological) laws of nature including lessons from new empirical evidence, (2.2) eliminating theoretical misrepresentation of the observable phenomena (e.g., artifacts like frame dependence) due to, for example, symmetry considerations, (2.3) resolving anomalies and inconsistencies within a theoretical framework, (2.4) identifying (causal) mechanisms, and (2.5) fully exploiting the explanatory resources of an established theory and expanding the realm of an established theory from within (second order generalisation). These strategies are internal in the sense that they are genuine to physics - that is strategies (2.1) to (2.5) constitute an essential and basic part of everyday physics research with its minimal set of aims - and do not appeal to metaphysical or metatheoretical (i.e., external) reasoning. 
To construct (or find) laws of nature as inductive generalisations is widely agreed to be a genuine and basic part of physics. Inductive generalisations are at the very basis of physics, and hence in some sense the starting point of unification, but they neither refer directly to unification (as mentioned above, the standard for calling something 'unification' is typically higher), nor to (e.g., metaphysics-laden) considerations external to physics. ${ }^{11}$ But what about the other strategies? Do they refer directly to unification or to considerations external to physics? Do they involve some sort of metaphysical presupposition? As I will argue in more detail in the next sections, this is not the case. (2.2) merely involves a proper evaluation of the phenomena's representation in theory (e.g., regarding symmetry properties, where symmetry considerations simply account for the phenomena's independence of observational or theoretical artifacts); (2.3) appeals to the fundamental demand that theories need to be logically (or mathematically) consistent, but does not presuppose anything about the comprehensiveness of the theory; (2.4) is a paradigmatical method of physics that does not involve any claim regarding the unity of such mechanisms; and (2.5) is an outstanding example of an internal unificatory practice as it simply comprises taking an empirically established theory seriously, fully exploiting it, and pushing it to its (alleged) limits by its own means (until anomalies arise)certainly, it is a genuine part of physics research to work out the details of its theories and check whether something has to give.

For further clarification, let me list two conceivable strategies that are - on their own - not genuine to physics, but refer to external reasoning: given two theories, (2.6) identifying a common (mathematical) structure or reducing one to the other, and (2.7) identifying a common ontology or reducing one to the other. Employing method (2.6) can be understood as enforcing the purely formal demand of mathematical simplicity on physics that merely reorganises the laws in a more 'simple' way (without additional explanatory insights). Moreover, (2.7) - if not a result of internal strategies - explicitly relies on philosophical considerations and does not seem to be a genuine physical method at all (physicists are typically not concerned with ontological questions in everyday research). ${ }^{12}$

\footnotetext{
${ }^{11}$ For a more detailed discussion see also section 3.1.

${ }^{12}$ One might object as follows: Why not say that ontological unification is a theoretical desideratum in physics? Since physical theories should be about the world, they should be about ontology. Ontological considerations would then, pace my own argument, be internal to physics: Often, genuine physical arguments just are arguments for ontological unification-I thank Isaac Wilhelm for raising this point. I think there are three issues here. First, I do not disagree that genuine physical arguments can result in ontological unification. I am arguing that this does not imply that ontological considerations are themselves part of the genuine methodology of physics. Second, I take it that most physicists (and philosophers thinking that physics is about empirical adequacy, for example) would not be on board with saying that ontological considerations are part of physics. Physics certainly is about the world, but it is specifically about the empirical (observable) aspects of the world. Third, whether ontological considerations are internal to physics or not is irrelevant with respect to the question of how to explain the unificatory practice: Claiming that ontological considerations are internal to physics is not sufficient to account for ontological unification. Being about ontology is not synonymous with being about a unified ontology. The real job (to answer why ontological considerations lead to a unified ontology) still remains to be done. For this we can again either appeal to external, i.e., philosophical reasons, or try to spell out internally why unification
} 
As already mentioned, some $d o$ think that physics employs externally justified strategies (see also section 3.4).

Note that these strategies are not isolated, but may overlap or work together. For example, an analysis according to (2.1) inductive generalisation or (2.2) eliminating misrepresentation can result in ontological unification. Also, I do not conceive the list of methodological strategies to be exhaustive or fixed, but as allowing for additional nuances. Furthermore, while (2.1) refers exclusively to (1.1), phenomenological unification, the other strategies might in principle account for unification at all three levels.

We can now identify the two concepts of unification, already mentioned, that are linked to a specific way of explaining why there is a unificatory practice in physics: The internal view holds that physics' genuine methodological strategies, (2.1) to (2.5), designed to uncover, exploit, and expand the explanatory resources of a given theory as far as possible, are sufficient to obtain unification at all three levels, (1.1) to (1.3), as a by-product of physics research. Because of its internal unificatory impact, we should not be surprised that genuine physics methodology on its own already implies a unificatory practice. Such a view neither draws on metaphysical or metatheoretical considerations, nor on explicitly aiming at unification. Accordingly, unification arises in physics. ${ }^{13}$

On the other hand, the external view argues either that we pursue unification for its own sake (or as being instrumental for promoting other epistemic values, like explanation ${ }^{14}$ for example), or that we pursue unification based on metaphysical or metatheoretical presuppositions. At the heart of both lies a unificationist methodology that executes the external demands that drive the striving for unification; strategies (2.6) and (2.7) may serve as examples of how physics' genuine methodology could be extended in the direction of a unificationist methodology. According to the external view, unification is imposed on physics. Hence, unification is a result of additionally constraining research by assumptions external to physics proper. The reasoning essentially works as follows: 'Due to some metaphysical or metatheoretical presupposition we know that physics should pursue unification. Therefore, we try to find (and should prefer) common theoretical and ontic structures to construct unified theories.' For instance, the way Maudlin presents the physicist's tale as a quest for (1.3) a unified ontology of the fundamental interactions suggests that physics pursues an externally justified unificationist methodology by explicitly trying to (2.7)

is the result of genuine physical arguments. This is precisely what I put forward from the outset: Often, genuine physical arguments turn out to promote ontological unification (as a by-product).

${ }^{13}$ At first sight, Maudlin (1996) seems to endorse an internal view when he argues that it is possible to identify empirical or theoretical evidence suggesting unification in many cases. However, Maudlin's conviction that physics is commited to unification as "the aim of physical theory" (Maudlin, 1996, 129f), his presentation of the physicist's tale, and his interpretation of certain case studies (see section 3.2, for example) are incompatible with the internal perspective $I$ advocate in this paper and rather point to a position that views physics as constrained and guided externally.

${ }^{14}$ In this paper I shall remain neutral on the issue of whether there is any conceptual link between unification and explanation. In general, I conceive my position on unification as compatible with views on which physics aims at explanation or truth. 
identify a common ontology. Proponents of this view emphasise that physicists are - and should be - dissatisfied with any kind of patchwork in physics (e.g., two completely disconnected theories for different interactions) for the respective reasons. Critics take this practice as de facto existing but misguided, and the reasons as physically dubious.

This paper argues against both perspectives: there is no such practice of imposition, but still unification is obtained. We should not interpret physics practice in line with the external view, because it obfuscates how theory development is actually driven and justified in physics, namely by concrete physical reasons. Remarks by physicists on their intentions or motivations should not be taken too seriously. Instead of evaluating what physicists say, I propose to look at what they actually do.

\section{Four Case Studies From Physics}

To support my claim that the practice of unification in physics does not rely on metaphysical or metatheoretical presuppositions imposed on physics, but is best explained by the genuine methodological practice of physics intended to uncover and exploit the explanatory resources of a given theory by promoting internal theoretical progress, I will now investigate four paradigmatic case studies.

\subsection{Newton's Universal Law of Gravitation}

The historically first substantial example of unification in physics is Newton's universal law of gravitation which famously unified the terrestrial and celestial mechanics of Galileo's and Kepler's laws. Equally famously, Newton was aware of and explicit about the metaphysical foundations and consequences. So, does this provide an instance where unification was driven by metaphysical reasoning?

Reading Ducheyne (2005), for example, it would seem so. Ducheyne argues that we can identify two types of unification in Newton's work. The first type of unification is established by identifying causal mechanisms, while the second refers to inductive generalisations. Ducheyne specifies that the first type "is based on the premise that nature maintains the same modus operandi as much as possible", whereas the second "is based on the premise that nature is contiguous" (Ducheyne, 2005, 77). Regarding the first type of unification,

[...] Newton infers from the observation that Kepler's second and third law hold for the primary and secondary planets that these motions are caused by inverse-square centripetal forces. [...] Newton concludes that since these phenomena are of the same kind they depend on causes of the same kind [...]. (Ducheyne, 2005, 74)

Ducheyne goes on to argue that after Newton has identified gravity as the common cause for planetary motion, "a deep belief in the contiguity of nature" (Ducheyne, 2005, 74) fuels the subsequent development towards his universal law of gravitation. 
We could understand this as follows: There are two metaphysical assumptions in Newton's work. On the one hand, nature is assumed to 'maintain the same modus operandi', on the other hand, nature is assumed to be 'contiguous'. Unification is then obtained by help of these assumptions. For example, unification by means of method (2.4) - identifying causal mechanisms - is based on the metaphysical assumption that nature 'maintains the same modus operandi', i.e. the same effects have the same cause. Unification would then depend on imposing metaphysical assumptions, just as the external view has it.

Against this, I propose to take the assumption that the same effects have the same cause as a general methodological (not a metaphysical) point about how inductive generalisation needs to be approached. If all the empirical facts about some set of phenomena suggest that they are (empirically) 'of the same kind', then science needs to take this seriously. Similarly, 'having the same cause' is just the best explanation for 'having the same effect'. If all facts speak in favour of uniformity and generalisability, it would seem dubious, if science still tried to keep things apart and told an elaborate story of why 'what is really going on' is more complex than the phenomena reveal. ${ }^{15}$ In brief, there are good internal reasons for developing a more unified description, and they do not commit us to any substantial metaphysical claim.

But even a stronger reading that accepts Newton's assumptions as metaphysical (i.e., as assumptions about the world, not about physics' methodology), does not entail that the external view is correct. This is because both assumptions do not selectively guide and drive the specific endeavour of unification (e.g., unification of two already established laws of nature), but are of concern in a more fundamental way: they are part of what allows us to engage in science (or inductive generalisation) in the first place. In this sense I agree that, for example, 'same effect, same cause' is a credo paradigmatically internal to physics. ${ }^{16}$ Some "order in the world" (Mumford and Tugby, 2013, 11) just has to be assumed; if you wish you may take this as an almost transcendental argument on the conditions of doing science per se (e.g., Mumford and Tugby (2013)). Obviously, this does have some impact in terms of initiating unification, but it does not imply that physics additionally employs metaphysical reasoning in order to specifically account for unification.

Let me also argue in more detail why the contiguousness assumption should count as internal to physics: I propose to read this assumption as an example of internally promoting a continuity from one theory (or phenomenological realm) to another allegedly disconnected theory (or phenomenological realm) - in the sense that a theory is taken seriously and then pushed to its limits by its own means as far as feasible. To demonstrate the continuity between terrestrial and celestial mechanics (which hitherto had been thought to be strictly separated realms), Newton brings up the following thought experiment in his The System of the World (see Newton (1846)): ${ }^{17}$ Suppose we are on a high mountain and

\footnotetext{
${ }^{15}$ See also the discussion of Einstein's dissatisfaction with Maxwell's theory of electromagnetism in the next section.

${ }^{16}$ I thank Isaac Wilhelm for pressing me on this.

${ }^{17}$ Note that the 'Moon-test' (Principia, Book III, Proposition IV) provides a more precise,
} 
drop a stone so that the stone freely falls to the ground. This is described by Galileo's terrestrial mechanics. Now, suppose we do not let the stone fall, but throw it. Terrestrial mechanics tells us that the stone will move on a parabolic trajectory. When we increase the velocity (parallel to the earth's surface) with which the stone is projected, the stone goes farther and farther. At some point the velocity is high enough to keep the stone from falling. The stone is now orbiting the earth just as a satellite is according to Kepler's laws. By means of taking seriously the theoretical framework and realm of Galileian physics and literally pushing it to its limits (the stone orbiting is obtained as a limiting procedure of ballistic trajectories), we end up in the framework of Kepler, suggesting an underlying unified description of both; and, in particular, suggesting an underlying mechanism or common cause. Thus, Newton's unification of celestial and terrestrial mechanics is best explained by the internal unificatory impact of physics' genuine methods and can be classified as unification at all three levels (phenomena, theoretical structure, and ontology) by means of a mixture of the different internal strategies of physics.

Before we move on, let me point out that Newton's unification is at least slightly different from the following examples as it is heavily concerned with an analysis of the phenomena, whereas the following examples will more and more prominently involve theoretical considerations. We may attribute this to the fact that the different examples have different points of departure regarding the already obtained level of unification. This may also explain why, as discussed, Newton's rules of reasoning more directly touch on fundamental aspects of scientific methodology than the following examples do.

\subsection{Electromagnetism in Special Relativity}

According to my scheme, Maxwell's theory exemplifies the phenomenological and theoretical unification of electricity and magnetism. In SR this is pushed further to ontological unification by eliminating electric and magnetic fields from the ontology and replacing them with the electromagnetic field. But which practice of unification was at play here? Maudlin argues that Einstein identified the lack of ontological unification in Maxwell's theory as defective and explicitly aimed at unifying electricity and magnetism. He interprets Einstein's appraisal of the moving magnet and conductor problem as straightforwardly concerned with unification:

$[\ldots]$ the failure of classical electromagnetic theory to unify electric and magnetic phenomena was the leading complaint voiced in Einstein's "special relativity" paper. (Maudlin, 1996, 131)

Thus, it seems that the transition from Maxwell's theory of electromagnetism to

quantitative argument based on empirical data for the fact that "the force by which the moon is retained in its orbit is that very same force which we commonly call gravity; for, were gravity another force different from that, then bodies descending to the earth with the joint impulse of both forces would fall with a double velocity [...]; altogether against experience" (Newton, 1846). 
$\mathrm{SR}$ is best explained by a unificationist methodology. Yet, in fact, "the failure to unify" was not Einstein's complaint. Reading Maudlin, one could come away with the impression that Einstein had no physical reasons to be dissatisfied with Maxwell's theory, but took a unified ontology to be better for other reasons external to physics. Reading Einstein, however, we find that he was tackling a concrete physical problem and obtained unification as the result of a genuine physical argument. Exploiting his principle of relativity, Einstein noticed that "Maxwell's electrodynamics [...] when applied to moving bodies, leads to asymmetries which do not appear to be inherent in the phenomena" (Einstein (1952) in Maudlin (1996)). The same phenomena generated by the same observable current in the conductor are interpreted differently (inconsistently) in Maxwell's theory: the charges in the conductor experience either a magnetic or an electric force depending on which frame of reference is chosen (the magnet frame or the conductor frame, respectively). Thus, the actual phenomenon is misrepresented in Maxwell's theory:

The observable phenomenon here depends only on the relative motion of the conductor and the magnet, whereas the customary view draws a sharp distinction between the two cases in which either one or the other of these bodies is in motion. (Einstein (1952) in Maudlin (1996))

So, Maxwell's theory artificially distinguishes two cases for one and the same $o b$ servable phenomenon (due to frame-dependent artifacts). Einstein argues that either there is a physical explanation of the asymmetries present in Maxwell's theory (that may also be tested empirically), or the theory suffers from framedependent artifacts (without observable consequences) hinting at some underlying structure that is invariant under change of reference frames. Through analysing Maxwell's theory, identifying misrepresentations of phenomena, and eliminating them in SR, Einstein arrived at an empirically more adequate description and thereby obtained unification. Why consider this to be a case of 'aiming at unification' when it was about attacking a theoretical misrepresentation of a phenomenon? Just because unification was the result? For Einstein, Maxwell's theory was not defective due to a failure of unification, but due to the fact that Maxwell's theory did not consistently explain the phenomena: specifically, it did not explain why the physical situation should be different when we change the reference frame (the underlying reason for this inconsistency being that the forces present transform differently than the fields giving rise to these forces). This is important to emphasise: it is a concrete physical problem (the moving magnet and conductor problem) that raises these questions, not ideas about how Newtonian and Maxwellian physics or electric and magnetic fields have to be unified. In other words, there is something to learn physically, which then promotes theoretical progress. The resulting unification was not based on any reference to external (metaphysical or metatheoretical) arguments, but was obtained by means of methods like (2.2) analysing and eliminating theoretical 
misrepresentation of the observable phenomena. ${ }^{18}$

There are certainly more instances of unification concerning SR. For example, joining space and time to obtain 'spacetime' is again a mere - though significant-by-product of getting the symmetry group right. But instead of investigating SR more thoroughly, let me now turn to a more controversial example from particle physics.

\subsection{Electroweak Theory and Grand Unified Theories}

As the physicist's tale illustrates, many are convinced that physics, among other things, tries to unify the three Standard Model (SM) interactions (electromagnetic, weak, and strong). Sheldon Glashow, Abdus Salam, and Steven Weinberg managed to accomplish the first step by presenting a unified theory for electromagnetism and the weak interaction. However, electroweak theory is usually not understood as exhibiting ontological unification, but rather seems to approach unification as an issue of formal simplicity without explanatory benefit (see Morrison (2000)). Furthermore, all attempts at grand unified theories (GUTs) which encompass all three SM interactions failed. Naturally, the suspicion arises that unification is not being pursued for internal reasons of physics here, but to meet external criteria. This is why studying the cases of electroweak theory and GUTs is interesting for my objective. It turns out that, for example, to formulate a consistent and empirically adequate theory of the weak interaction in the first place, we needed to include the electromagnetic interaction (Maudlin, 1996, 138). So, unification was the (surprising) result of constructing a theory encompassing the phenomena of the weak interaction. I will get back to this in a moment, but let us approach the matter step by step. First of all, what does 'unifying interactions of the SM' mean? Let me briefly recall how these interactions are represented in theory.

The quantum field theories (QFTs) of the SM are so-called gauge theories. In short, the SM interactions are represented by gauge fields that arise according to the group generators of some underlying symmetry group. So, a gauge theory is essentially defined by its symmetry group. Unifying interactions then amounts to group-theoretic operations. Basically there are three different types.

First of all, two (or more) symmetry groups can be 'glued' together to form a so-called product group. The product group is a trivial mathematical combination of different groups, which can always be constructed. The SM is put together like this: the symmetry groups of the electromagnetic, U(1), the weak, $\mathrm{SU}(2)$, and the strong interaction, $\mathrm{SU}(3)$, are merged by a trivial mathematical operation: $\mathrm{SU}(3) \times \mathrm{SU}(2) \times \mathrm{U}(1)$. In Maudlin's terms "this is a case of common dynamics and nothing more" (Maudlin, 1996, 137). It "does not constitute any

\footnotetext{
${ }^{18}$ Let me briefly comment on a worry that was similarly raised to me by Andreas Hüttemann and Isaac Wilhelm. One could ask: what is so bad about an imperfect representation? What is so bad about having symmetric explananda, but asymmetric explanantia? Implicit in the question is the suggestion that 'aiming at unification' is the answer. I do not see how aiming at a correct representation of phenomena involves ideas on unification. In some cases it may imply unification as a result; but this is not what I am arguing against, but arguing for.
} 
sort of unification of the theories at all" (Maudlin, 1996, 139). We do not learn anything above and beyond what we already knew. However, second, unification by means of a product group may reveal additional structure. Consider, for example, electroweak theory. The unified gauge group is just the product group $\mathrm{SU}(2) \times \mathrm{U}(1)$, but a nontrivial mixing between the generators of the symmetry groups occurs when combined to form the product group. Hence, the combination seems to be less superficial. Third, gauge theories can be unified by combining symmetry groups into a so-called simple group. This is not a trivial mathematical operation. One cannot simply join some groups to form a simple group. At least regarding mathematical structure, unification via some simple group is more substantial than via a product group. Physically, there are also differences. For example, an adequate choice for a simple group unifying the SM usually involves additional physically relevant structure. So we might argue that we have physical reasons to pursue such proposals. Consider, for example, $\mathrm{SU}(5) \supset \mathrm{SU}(3) \times \mathrm{SU}(2) \times \mathrm{U}(1)$ as an approach to GUTs. All such attempts at GUTs have failed due to the lack of empirical adequacy. Most prominently, GUTs predict a half-life for proton decay that is empirically excluded.

What drives these attempts at unification? Is it unification for the sake of formal simplicity? For electroweak theory we find this not to be the case. Rather, electroweak theory was not intended to be an attempt at unification at all. While we can write down a theory of electromagnetism alone, as obtained with quantum electrodynamics, we cannot write down a theory of the weak interaction only: ${ }^{19}$

No workable theory of the weak force existed before the unified theory $[\ldots]$ Incidentally, this is one way in which the usual story about unifying forces is wrong. It is not that at some point we had theories of the electromagnetic, weak, strong, and gravitational forces separately, and now we have managed to unify the first two. Rather, at some point we recognized the existence of all four forces, and found that unification was needed to account for the weak force. (Maudlin, 1996, 141)

So, electroweak theory was obtained as a by-product of constructing an empirically adequate theory of the weak interaction, and hence proves to be an example of physics' internal unificatory practice; notably, although it is typically argued that "the unification is partial at best" (Georgi in Maudlin (1996)): electroweak theory neither uncovers a causal mechanism, nor provides ontological unification, nor exhibits any particular explanatory power beyond merely accounting for the weak force, but solely unifies mathematical structure ( $c f$. Georgi in Maudlin (1996), and Morrison (2000)). In my scheme, electroweak

\footnotetext{
${ }^{19}$ Let me stress that, strictly speaking, this presentation adopted from Maudlin (1996) is somewhat imprecise: we did know not only some phenomenological details about the weak interaction, but also already had some effective theoretical modelling; I thank Sébastien Rivat for reminding me of this. Still, it is correct to say that electroweak unification was not about unifying two equally accepted and well-behaved theories. A sensible theory of the weak interaction was not worked out yet.
} 
theory is therefore classified as unification of types (1.1) and (1.2), namely concerning phenomena and theoretical structure, by means of different internal strategies of physics, this being some mixture of types (2.1) to (2.5).

What about GUTs? Here, the problem seems to be different. We already have a worked out theory for the strong interaction: quantum chromodynamics. Why unify two already existing theories? It might seem we can only make sense out of this research programme by referring to external reasoning or at least have to acknowledge that this direction of research was pursued for heuristical reasons. However, a closer look reveals that GUTs were not considered for purely heuristical, but for internal physical reasons. Due to the mathematical structure of these theories, physicists anticipated that GUTs would yield additional information for electroweak theory with higher explanatory power:

The motivation for the simplest GUT, SU(5), was not any mystical desire to follow in Einstein's footsteps and unify everything. Shelley Glashow and I were just trying to understand $\mathrm{SU}(2) \times \mathrm{U}(1)$ better. For several years, we had realized that if we could incorporate the $\mathrm{SU}(2) \times \mathrm{U}(1)$ gauge symmetry into a single simple group it would give us some extra information. It would fix the value of the weak mixing angle, a free parameter in the $\mathrm{SU}(2) \times \mathrm{U}(1)$ theory and it would explain why all the electric charges we see in the world are multiples of the charge of the electron. (Georgi in Maudlin (1996))

Generally, such models are not analysed out of "mystical desires" for ontological unity or for the sake of formal simplicity, but to better understand the explanatory features and problems of an already established theory - by means of strategies (2.3), (2.4), and (2.5). Yet, bewilderingly, Maudlin summarises Georgi's comment - similar to how he read Einstein's comment on Maxwell's theory - by invoking what Georgi explicitly rejected: "these considerations all stem from the desire to complete the only partially unified $\mathrm{SU}(2) \times \mathrm{U}(1)$ theory in a more satisfactory way" (Maudlin, 1996,142). This underlines that Maudlin is not concerned with distinguishing whether unification is driven by internal or external reasoning.

\subsection{Quantum Gravity}

Such talk about physicists aiming at unification for (allegedly) physically dubious reasons is especially common and problematic when it comes to attempts at the frontier of physical research. Here, we often do not have much data to back new theoretical developments. Take QG, for example, which is usually presented as the quest to unify general relativity (GR) and QM (or QFT). ${ }^{20}$ When it comes to QG - the most prominent and most controversial case of unification in modern physics - we do not have any clear-cut, novel experimental or observational data at all. Additionally, the research programme of QG faces serious theoretical problems. According to folklore, this is because GR and QFT

\footnotetext{
${ }^{20}$ In fact, there is no consensus on what exactly the quest is.
} 
seem to exhibit a "fundamental incompatibility" (Maudlin, 1996, 143). So, why unify?

Most physicists and philosophers argue that there are many physical reasons to expect an underlying theory due to phenomena where both, GR and QFT, are understood to become relevant - among those are problems within the standard model of cosmology, black holes, and consistency issues in QFT (e.g., Kiefer (2007)). Overall, these issues are taken to indicate some sort of incompleteness of present-day physics and trigger theoretical progress. Accordingly, the reason to pursue a theory of QG is to solve these genuinely physical issues - evidently an internal enterprise.

Critics, however, disagree and argue that there is in fact no compelling physical reason at all to pursue these research programmes - neither experimental, nor theoretical. Naturally, they advocate the disunitist's position of semiclassical theories (e.g., Mattingly (2005)). In the past, or so they argue, physics may have profited from (what they now consider a dubious) striving for unification, but modern research programmes like QG are misguided in their (alleged) commitment to unification. So have we finally encountered an example where unification is indeed driven by "purely aesthetic reasons", or "on the general methodological grounds of repeating strategies that succeeded in the past" arguments Maudlin already dismissed for GUTs as not convincing (Maudlin, 1996, 141)?

In fact, also philosophers and physicists who generally accept the need for a theory of QG frequently mention 'aiming at unification' or a 'desire for unification' as an additional reason (e.g., Kiefer (2007), Wüthrich (2005), and Crowther (2018)), and raise similar concerns - at least regarding the motivation to pursue specific approaches, e.g. in the context of particle physics (e.g., Hossenfelder (2018)). For instance, Wüthrich argues that

A strong, but nevertheless often nebulous, desire to present a unified theoretical framework at the level of fundamental physics populates the folklore of physicists and often fuels the search for a quantum theory of gravity. Arguments to this effect, relying - if made explicit at all — on metaphysical considerations, typically elicit some principles of unity of nature or of scientific method. Although general relativity and quantum theory may be so disparate as to disallow the formal deduction of contradictions, they are generally taken to be incommensurable (families of) theories. A quantum theory of gravity is expected to remedy this theoretical schism and to bolster attempts at finding the Holy Grail of physics, a unified framework of all interactions. The argument from unification - unification for the sake of unification - does not, however, sway the sceptic. The "disunitist" would certainly be free to respond that at the very least, it may just as well be the case that the conceptual disunity of the two theories reflects a disunity in nature. In fact, she could claim, gravity's stubborn refusal to be subsumed under the otherwise allencompassing umbrella of the Standard Model may be interpreted 
as evidence for this disunity. Despite its rare explicit articulation and its questionable metaphysical strength, however, the unificatory impetus provides an extremely important motivation for attempts at quantizing gravity. (Wüthrich, 2005, 788)

Pushing Wüthrich's worry further, Mattingly (2005) is convinced that "implicit as well as explicit philosophical motivations" and "meta-theoretical commitments of some kind" drive the research programme. In order to support his claim, Mattingly refers to the following statement by Carlo Rovelli:

We have learned from GR that spacetime is a dynamical field among others, obeying dynamical equations, and having independent degrees of freedom: a gravitational wave is extremely similar to an electromagnetic wave. We have learned from QM that every dynamical object has quantum properties, which can be captured by appropriately formulating its dynamical theory within the general scheme of QM. Therefore, spacetime itself must exhibit quantum properties. Its properties - including the metrical properties it defines - must be represented in quantum mechanical terms. Notice that the strength of this "therefore" derives from the confidence we have in the two theories, QM and GR. (Rovelli, 2001)

Does this reveal that Rovelli uses "some kind of thesis about the unity of nature" and that "an important meta-theoretical impetus for quantising gravity follows from notions of unification", as Mattingly (2005) argues? Rovelli's verdict regarding the 'extreme similarity' and its relevance arguably does seem to express some metaphysical presupposition, but had better be read as being about comparing the theoretical concepts of two theories, and then drawing conclusions about the world. Accordingly, some (metaphysically more modest) story in line with Kao (2017) better accounts for what is going on here. Still, Rovelli's statement on the lessons from QM begs the question, because the level of generality ("every dynamical object") is chosen with respect to the desired (rhetorical) argument in favour of unification. So, on the face of it, the critics seem to have a point.

However, philosophy should not get distracted by programmatic remarks. What is crucial is an evaluation of the actual practice of physics. Due to the multitude of different approaches this cannot be done exhaustively for all QG research programmes in this paper. Instead I will focus on a particular class of approaches in the context of particle physics and string theory that is - in line with the physicist's tale often thought to be specifically about a striving for unification. ${ }^{21}$ In the following, I will argue that we can make Rovelli's remarks more precise in this context and thereby rebut the critics who do not accept the mentioned physical reasons to engage in the research programme of QG. This is done by emphasising the substantive insights from QFT.

\footnotetext{
${ }^{21}$ Alternative approaches to QG usually have a rather different and less comprehensive objective. Loop quantum gravity (LQG), for example, is merely concerned with a quantum theory of (empty) spacetime.
} 
As argued in Salimkhani (2018) — summarising the standard view of particle physics on this issue based on the work of Weinberg and others - a close analysis of the framework of QFT delivers an effective theory of quantum gravity that is empirically adequate, but has specific theoretical problems that need to be resolved. In particular, Lorentz-invariant interactions with massless spin-2 particles require the equivalence principle to hold at low energies (e.g., Weinberg (1995)) which results in a low-energy theory of quantum gravity that has GR as its unique classical limit - this is a working theory of QG. However, the theory becomes inconsistent in the most interesting regime, namely at high energies (the infamous renormalisation problem). ${ }^{22}$ Not only does QFT provide us with a first working theory of QG, but also shows that we need to go beyond. Note also that GR is of no concern - we may actually assume that we did not know about GR. As a result, the issue of QG is not to unify two theories of allegedly separate realms (GR and QFT), but to fix internal issues of QFT, i.e. to find the correct high-energy theory.

In a first step, Weinberg's argumentation establishes a theory of QG. This is "an example of physics' internal capacity to promote theoretical progress by fully exploiting an existing theoretical framework and thereby uncovering substantial links to allegedly disconnected phenomena and theoretical structures" (Salimkhani, 2018), i.e., strategy (2.5). Taking the best theoretical framework seriously, and analysing it thoroughly, results in a low-energy theory of QG. In a second step, we then find that QFT does not provide a meaningful theory of gravity at high energies. This is the remaining problem of QG that needs to be resolved for purely internal reasons (namely for reasons of consistency of our best theory: QFT). This amounts to pursuing strategy (2.3). In fact, closely related live approaches like asymptotic safety and string theory (e.g., Weinberg (1999) and Dawid (2013)) are the result of such an analysis of QFT, and may also help to better understand QFT. ${ }^{23}$

\section{Unification revisited}

Let us now briefly retrace our steps. We started by observing that over time the theories of physics have become more and more unified, and that this calls for an explanation. We noticed that unification being the ultimate aim of physics (or being instrumental for other epistemic values) is what many would accept as an explanation, although it is unclear what exactly this implies methodologically. Furthermore, for some (e.g., Mattingly (2005) and Hossenfelder (2018)) the genuine methodology of physics, a tendency towards unified theories in the past, and previous success of certain heuristic strategies are not able to justify present research programmes - be it in principle or as a matter of fact. In

\footnotetext{
${ }^{22}$ Let me add that I am not suggesting that a theory is 'correct' if and only if it is UVcomplete. Crowther and Linnemann (2018) nicely evaluate this issue.

${ }^{23}$ Note that the internal-external distinction may also be used to classify approaches as more or less promising. Considering the amount of disagreement between the different approaches to QG it should come as no surprise if certain approaches eventually become viewed as poorly justified.
} 
this perspective, especially certain research programmes at the frontier which (if successful) promise unification as one of their key features look suspiciously like a misguided striving for unification - in the specific sense that physicists either naively try to repeat previous successes or start from metaphysical or metatheoretical presuppositions which turn out to severely constrain (and potentially mislead) their research. The critics' view, in short, is that unification is 'imposed' on physics (for external and potentially dubious reasons).

Against this, I emphasised that a more rigorous study of the actual practice of unification in physics reveals that unification arises from genuine methods of physics alone. Unification does not depend on metaphysical presuppositions, but is the result of good scientific practice. Unification is best explained by a certain set of basic methods used in everyday physics. More specifically, unification is best explained by the practice of taking a concept or a theory seriously, thoroughly working out its details, pushing it to its limits, and thereby gaining new knowledge. ${ }^{24}$ This is the 'best' explanation because it explains the unificatory practice from within physics (without assuming additional or controversial aims and methods). Furthermore, the opposing position (1) would have to say how exactly physics methodologically incorporates the alleged external constraints (either this will involve implausible statements like 'physics is directly concerned with working out a minimal ontology' or it effectively collapses into my proposal), (2) cannot provide any explanatory benefit which could justify their additional metaphysical assumptions, and (3) obfuscates the actual practice of physics.

In this respect, my project is that of reducing the share of metaphysical (or metatheoretical) presuppositions in explaining actual practice in physics. Note that my project is not that of arguing against any metaphysical basis (or formal constraints) for scientific endeavours: the position I propose does still allow for physics making fundamental assumptions about its objects, in the sense that physics may have to assume that the objects are well-behaved enough to be objects of physical research in the first place. Regarding unification, however, metaphysics is not what we start with but where we end up: it is the (inductive) result of physics (depending on it and being continuous with it). So even if those methods I take to be genuine to physics are not metaphysically thin but loaded with some basic (metaphysical) assumptions, we are still better off not viewing unification as driven by metaphysical or metatheoretical presuppositions. Note that we may say that the basic assumptions actually do the work, but this is still different from what Wüthrich (2005) and Mattingly (2005) put forward, for example.

There is another important aspect to my presentation: Arguing that unification will come out as a result of proper research implies a certain sense of necessity. Within a particular line of thought (concept or theory), the tendency towards a more unified picture is inevitable - at least up to the point where

\footnotetext{
${ }^{24}$ This is not only characteristic of physics, but of mathematics as well. Consider the example of number theory where exploiting the notion of 'number' further and further ('pushed to its limits') ultimately provided substantial links to other areas of mathematics. I thank Andreas Bartels for this.
} 
the concept or theory breaks down and is not useful anymore. This view is to be distinguished from, for example, a 'best systematisation' view that can only make sense of unification in terms of a strong and explicit dependence on external considerations regarding simplicity, for example.

Apart from lessons in general philosophy of science, this is an interesting result for metaphysics as well, especially for programmes that employ abductive reasoning. So, let me briefly comment on how arguing that unification in physics does not depend on metaphysical or metatheoretical presuppositions may be exploited further for attacking the selection problem in inductive (and in naturalised) metaphysics, for example.

First, let me clarify that by inductive metaphysics (IM) I do not refer to the historical programme of Becher and others, but a recent approach to metaphysics similar to Williamson (2016), for example. Modern IM asserts a specific methodological continuity between science and metaphysics. It holds that (inductive) metaphysical inferences should not only meet scientific facts, but (a) employ scientific facts as (empirical) premises and (b) proceed in accordance with scientific methodology by using, for example, inferences to the best explanation. As a result, metaphysical generalisation is proposed to be not categorically different from science, but rather to collapse into (more general) scientific generalisation. In particular, metaphysical theories are taken to be contingent (in the sense that they depend on certain scientific theories). Also, note that IM takes seriously that we should not read off metaphysical commitments from a particular scientific theory alone - as naturalised metaphysics may be charged with - but also consider available alternative theories (or alternative interpretations, for that matter).

Now, since the ontological commitments of the respective theories may differ substantially (e.g., see the approaches to QG), it seems that we cannot hope for a unique determination of any positive metaphysical inference unless we ultimately appeal to what is usually referred to as 'a priori reasoning' by help of assumptions like simplicity or unification ( $c f$. Paul (2012)). Otherwise, we might only be able to carve out a 'common ground' as a merely negative constraint on metaphysics. In fact, IM does not generally oppose such elements of a priori reasoning, precisely for the reason that it wants to be able to further constrain inductive metaphysical inferences. However, one may argue that IM is rendered unoriginal by taking this 'loophole'.

It is here where an internal understanding of unification is relevant for inductive metaphysics in two respects. First, the internal view explicates how inductive methods of physics bring about decisions for ontology not only by underlining some continuity between everyday physical research and metaphysics, but also by proposing that metaphysics itself should be understood inductively. Second, showing that unification is internal in the sense presented provides a cure to the mentioned problems in metaphysics in terms of a selection rule for choosing which scientific theories should be considered relevant for (inductive) metaphysical inferences: namely, choose the theories that are 'best' with respect to unification. An internal conception of unification can thus help to diminish worries about using such criteria, since it better meets IM's general 
line of thought than the canonical (external) take on unification. Accordingly, we may employ unification as a positive constraint to inform IM about which physical theories to consider without this meaning that IM is forced to embrace a priori reasoning after all.

Besides this, one could also straightforwardly argue that the described practice of physics allows for the following (inductive) metaphysical inference: there actually is a unity in nature (at least to a certain extent) because physics internally obtains unification (at least to a certain extent) as a result of inductive scientific reasoning. ${ }^{25}$

Acknowledgements. I especially thank Andreas Bartels, Karen Crowther, Andreas Hüttemann, Rasmus Jaksland, Niels Linnemann, Isaac Wilhelm, and two anonymous reviewers for very helpful feedback on earlier versions of this paper. I am also grateful to Claus Beisbart, Stefan Heidl, Niels Martens, Sébastien Rivat, Matthias Rolffs, and Ben Young for useful discussions and remarks. Furthermore, I would like to thank the audiences at the Frühjahrstagung der Deutschen Physikalischen Gesellschaft in Hamburg in February 2016, the 9th European Congress of Analytic Philosophy in Munich in August 2017, the XXIV. Kongress der Deutschen Gesellschaft für Philosophie in Berlin in September 2017, and the 3rd Annual Conference of the Society for the Metaphysics of Science in New York City in October 2017 for valuable comments.

\section{References}

Cartwright, N. (1999), The Dappled World, Cambridge University Press.

Cat, J. (1998), 'The physicists' debates on unification in physics at the end of the 20th century', Historical Studies in the Physical and Biological Sciences 28(Part 2), 253-300.

Crowther, K. (2018), 'Defining a crisis: the roles of principles in the search for a theory of quantum gravity', Synthese .

Crowther, K. and Linnemann, N. (2018), 'Renormalizability, fundamentality, and a final theory: The role of UV-completion in the search for quantum gravity', British Journal for Philosophy of Science 0, 1-30.

Dawid, R. (2013), String Theory and the Scientific Method, Cambridge University Press.

Ducheyne, S. (2005), 'Newton's notion and practice of unification', Stud. Hist. Phil. Sci. 36, 61-78.

Einstein, A. (1952), On the Electrodynamics of Moving Bodies, in 'The Principle of Relativity', New York: Dover.

\footnotetext{
${ }^{25}$ To be clear, this means that if there were two perfectly fine, non-unifyable theories with no hints towards an encompassing framework, the same reasoning would suggest a disunitist position.
} 
Friedman, M. (1974), 'Explanation and Scientific Understanding', The Journal of Philosophy 71(1), 5-19.

Hüttemann, A. (1997), Idealisierungen und das Ziel der Physik. Eine Untersuchung zum Realismus, Empirismus und Konstruktivismus in der Wissenschaftstheorie, de Gruyter: Berlin, New York.

Hossenfelder, S. (2018), Lost in Math: How Beauty Leads Physics Astray, Basic Books.

Kao, M. (2017), 'Unification beyond justification: a strategy for theory development', Synthese pp. 1-16.

Kiefer, C. (2007), Quantum Gravity, Oxford University Press.

Kitcher, P. (1981), 'Explanatory unification', Philosophy of Science 48(4), 507531.

Mattingly, J. (2005), Is Quantum Gravity Necessary?, in A. J. Kox and J. Eisenstaedt, eds, 'The Universe of General Relativity', Birkhäuser, pp. 327-338. Talk delivered at the 5th International Conference on the History and Foundations of General Relativity in 1999.

Maudlin, T. (1996), 'On the Unification of Physics', The Journal of Philosophy 93(3), 129-144.

Morrison, M. (2000), Unifying Scientific Theories, Cambridge University Press.

Mumford, S. and Tugby, M. (2013), What is the metaphysics of science?, in S. Mumford and M. Tugby, eds, 'Metaphysics and Science', Oxford University Press, pp. 3-26.

Newton, I. (1846), The Mathematical Principles of Natural Philosophy, Daniel Adee, New York. translated by Andrew Motte; includes Newton's System of the World.

Paul, L. A. (2012), 'Metaphysics as modeling: the handmaiden's tale', Philos Stud 160, 1-29.

Rickles, D. and French, S. (2006), Quantum gravity meets structuralism: Interweaving relations in the foundations of physics, in D. Rickles, S. French and J. Saatsi, eds, 'The Structural Foundations of Quantum Gravity', Oxford University Press, pp. 1-39.

Rovelli, C. (2001), Quantum spacetime: What do we know?, in N. Huggett and C. Callender, eds, 'Physics meets philosophy at the Planck scale. Contemporary theories in quantum gravity', Cambridge University Press, pp. 101-122.

Salimkhani, K. (2018), Quantum gravity: A dogma of unification?, in A. Christian, D. Hommen, N. Retzlaff and G. Schurz, eds, 'Philosophy of Science. European Studies in Philosophy of Science, Vol 9.', Cham: Springer, pp. 2341. 
Salmon, W. C. (1984), Scientific Explanation and the Causal Structure of the World, Princeton: Princeton University Press.

Salmon, W. C. (1990), 'Scientific Explanation: Causation and Unification', Crítica: Revista Hispanoamericana de Filosofía 22(66), 3-23.

Steinberger, F. (2017), The normative status of logic, in E. N. Zalta, ed., 'The Stanford Encyclopedia of Philosophy', spring 2017 edn, Metaphysics Research Lab, Stanford University.

Treder, H.-J. (1983), 'Was Gott getrennt hat, soll der Mensch nicht vereinen. Zum Problem der Großen Unitarisierung', Astronomische Nachrichten 304, 145-151.

von Weizsäcker, C. F. (1980), The Unity of Nature, Farrar, Straus, Giroux.

Wüthrich, C. (2005), 'To Quantize or Not to Quantize. Fact and Folklore in Quantum Gravity', Philosophy of Science 72, 777-788.

Weinberg, S. (1995), The Quantum Theory of Fields. Volume I: Foundations, Cambridge University Press.

Weinberg, S. (1999), What Is Quantum Field Theory, and What Did We Think It Is?, in T. Y. Cao, ed., 'Conceptual Foundations of Quantum Field Theory', Cambridge University Press, pp. 241-251.

Williamson, T. (2016), 'Abductive philosophy', The Philosophical Forum 47, 263-280. 